\title{
Política para Avaliação e Substituição de Frota por Meio da Adoção de Modelo Multicritério
}

Aray Gustavo Feldens
Mestrado em Engenharia de Produção pela Universidade Federal do Rio Grande do Sul

Praça Argentina, 9. 2 ${ }^{\circ}$ andar (LOPP). Porto Alegre/RS. CEP: 90040-020 E-mail:agfeldens@hotmail.com

Claudio José Muller

Doutorado Engenharia de Produção pela Universidade Federal do Rio Grande do Sul UFRGS

Professor da Universidade Federal do Rio Grande do Sul - UFRGS Av. Osvaldo Aranha, 99. 5ํaㄱ andar (UFRG/DEPT). Porto Alegre, RS. CEP: 90035-190 E-mail:cmuller@producao.ufrgs.br

Tiago Pascoal Filomena Doutorado em andamento na George Washington University - EUA Praça Argentina, 9. 2o andar (LOPP). Porto Alegre/RS. CEP: 90040-020 E-mail: tiagofilomena@gmail.com

Francisco José Kliemann Neto Doutorado em Engenharia de Produção - INP (França) Professor da Universidade Federal do Rio Grande do Sul - UFRGS Av. Osvaldo Aranha, 99. 5ํandar (UFRGS/DEPT). Porto Alegre/RS. CEP: 90035-190 E-mail:kliemann@producao.ufrgs.br

Antenor dos Santos Castro Mestrado em Engenharia de Produção pela Universidade Federal do Rio Grande do Sul Praça Argentina, 9. $2^{\circ}$ andar (LOPP), Porto Alegre/RS. CEP: 90040-020 E-mail: antenorcastro@yahoo.com.br

Michel José Anzanello Doutorado em Engenharia Industrial e de Sistemas - The State University of New Jersey Av. Osvaldo Aranha, 99. 5ำ andar (UFRGS/DEPT). Porto Alegre/RS. CEP: 90035-190 E-mail: anzanello@producao.ufrgs.com

\section{RESUMO}

O uso eficiente dos ativos fixos é um dos principais objetivos na administração de empresas do setor de transporte urbano. Os veículos de transporte determinam o nível de serviço oferecido e impactam significativamente na eficiência dos inputs envolvidos 
Política para Avaliação e Substituição de Frota por Meio da Adoção de Modelo Multicritério Aray Gustavo Feldens, Claudio José Muller, Tiago Pascoal Filomena, Francisco José Kliemann Neto,

no seu uso. O administrador pode otimizar a rentabilidade e o nível de serviço oferecido através da escolha do veículo apropriado e da definição da taxa de substituição do equipamento no tempo. Este artigo apresenta uma metodologia para a substituição de frotas de ônibus por meio da integração de critérios econômicos e não-econômicos. Aspectos econômicos são contemplados por indicadores econômicos e fluxo de caixa, utilizando custos de aquisição e manutenção da frota, entre outros. Aspectos nãoeconômicos são representados por decisões estratégicas e de gestão, sendo avaliados por intermédio de ferramentas de decisão múltipla. A metodologia proposta possibilitou avaliação detalhada do desempenho dos ônibus atuais frente a potenciais substitutos, viabilizando constante monitoramento dos ativos da empresa e assegurando elevados níveis de serviço ao cliente. A metodologia é ilustrada em uma empresa pública de transporte urbano.

Palavras-chave: Substituição de equipamentos. Análise multicritério. Transporte coletivo por ônibus.

\section{Multicriteria Fleet Evaluation and Replacement Policy}

\section{ABSTRACT}

The efficient management of companies' fixed assets has become a key issue to ensure stability on highly competitive markets. In urban transportation companies, these fixed assets represent a major share of the company's property and impact on the service level. This demands an efficient policy to evaluate fleet performance and replacement. This paper proposes a methodology for fleet evaluation and replacement by integrating economic and non-economic criteria. Economic aspects are evaluated by means of economic indices and cash flow of costs associated to acquisition and maintenance. Non-economic aspects are evaluated by strategic and operational attributes, and rely on multicriteria analysis tools. The proposed methodology allows performance comparison between current and alternative buses, enabling asset monitoring and assuring efficient service level. The methodology is illustrated in an urban public transportation company.

Key Words: Equipment replacement policy. Multicriteria analysis. Public bus transportation.

\section{INTRODUÇÃO}

O padrão de concorrência estabelecido, nos segmentos empresarias, demanda estratégias sólidas para a consolidação da organização. Este cenário exige a incorporação rápida e eficiente de novas tecnologias para atender às demandas do mercado externo, representado pelos consumidores, e interno, representado por funcionários e acionistas. A eficiente administração dos ativos fixos de uma empresa, 
Política para Avaliação e Substituição de Frota por Meio da Adoção de Modelo Multicritério Aray Gustavo Feldens, Claudio José Muller, Tiago Pascoal Filomena, Francisco José Kliemann Neto, Antenor dos Santos Castro, Michel José Anzanello

especialmente em termos de manutenção e reposição de componentes, figura entre os principais aspectos capazes de assegurar posição consolidada neste mercado. Em empresas do setor de transporte urbano, o uso eficiente dos ativos fixos está vinculado a uma política bem estruturada de avaliação e substituição de frota.

Alguns casos de substituição de frota aplicados ao segmento de ônibus urbano são reportados pela literatura especializada, como em Pinar e Hartman (2004), Khasnabis et al. (2002), Di e Hauke (2000) e Scarf e Bouamra (1999). No entanto, estes trabalhos estão fundamentados em dados simulados e/ou trabalham com formulações puramente teóricas. Dois tipos de modelos são normalmente sugeridos pela literatura para a substituição de frota: modelos de Engenharia Econômica (EE) e modelos de Pesquisa Operacional (PO).

Os modelos de EE restringem-se aos aspectos econômico-financeiros, considerando exógenas as variáveis de ordem tecnológica, de gestão e estratégica. Segundo Kaplan (1986), esta limitação dos métodos tradicionais leva a gerência a abandonar métodos formais de análise de investimentos e a utilizar análises subjetivas não-estruturadas. Essa realidade é observada por outros autores na literatura, como Carter (1992), Schinski (1999) e Collan e Liu (2003).

Já os modelos tradicionais de $\mathrm{PO}$, apesar de modelarem múltiplas variáveis, focam-se em um único objetivo a ser maximizado/minimizado. A adoção de um único critério limita aplicações práticas onde custos, eficiência e nível de serviço devem ser avaliados simultaneamente. Métodos como Multi Attribute Utility Theory (MAUT), Analytical Hierarchy Process (AHP), e Desdobramento da Função Qualidade (Quality Function Deployment - QFD) têm sido utilizados para acomodar múltiplos decisores e múltiplos critérios de decisão (ROY; VINCKE, 1983 EROL; FERRELL Jr., 2003, COLLAN; LIU, 2003). No entanto, não foram encontradas na literatura especializada abordagens consolidadas utilizando estes métodos na substituição de veículos de transporte coletivo.

O presente artigo apresenta uma metodologia consolidada para a substituição de frotas em uma empresa do setor de transporte coletivo urbano. A sistemática proposta inova na medida em que integra critérios econômicos e não-econômicos. Aspectos 
Política para Avaliação e Substituição de Frota por Meio da Adoção de Modelo Multicritério Aray Gustavo Feldens, Claudio José Muller, Tiago Pascoal Filomena, Francisco José Kliemann Neto, Antenor dos Santos Castro, Michel José Anzanello

econômicos são contemplados através do levantamento de custos associados à aquisição e manutenção da frota, entre outros, valendo-se de indicadores econômicos e fluxos de caixa. Aspectos não-econômicos contemplam aspectos estratégicos e de gestão por intermédio da integração dos métodos AHP, MAUT e QFD. O método proposto possibilita acomodar a opinião de múltiplos especialistas de processo (decisores), permitindo a avaliação do desempenho dos equipamentos atuais frente a potenciais substitutos, e servindo de suporte para decisões de aquisição de novos veículos. Outro aspecto a ser salientado está na flexibilidade do modelo, o qual pode ser facilmente adaptado à análise de outros ativos fixos.

A estrutura deste artigo é a seguinte: a seção 2 apresenta uma revisão teórica sobre os fundamentos econômicos dos modelos de substituição de veículos de transporte coletivo, bem como aspectos básicos dos modelos de decisão multicritério. A seção 3 traz uma descrição da metodologia proposta. A seção 4 ilustra os resultados da metodologia aplicada em uma empresa de transporte urbano. A conclusão encerra o artigo na seção 5. É importante salientar que este estudo está focado na apresentação do modelo sugerido. Os resultados numéricos são restritos aos valores conclusivos por limitações de espaço e aspectos estratégicos da empresa.

\section{REFERENCIAL TEÓRICO}

\subsection{Substituição de Equipamentos (SE)}

O conceito de Substituição de Equipamentos (SE) tem origem nos primórdios da Administração Científica. Os trabalhos pioneiros de Taylor (1923) e Hotelling (1925) utilizam como objetivo principal a maximização do Valor Presente de um único equipamento. Para isto, analisa-se o ciclo de vida do mesmo e determina-se a Vida Econômica - o ponto ótimo de substituição no período. Alchian (1958) sugere que os modelos de SE priorizam a lógica de otimização econômica, ou seja, os equipamentos são substituídos quando o valor econômico do equipamento novo (custo inferior ou lucro superior) é superior ao apresentado pelo equipamento atual.

Segundo Fleischer (1973), o Sistema MAPI, desenvolvido por George Terborgh, permite determinar a política de substituição com base em um 'gradiente de 
Política para Avaliação e Substituição de Frota por Meio da Adoção de Modelo Multicritério Aray Gustavo Feldens, Claudio José Muller, Tiago Pascoal Filomena, Francisco José Kliemann Neto, Antenor dos Santos Castro, Michel José Anzanello

inferioridade'. Tal sistema avalia decréscimos nos indicadores de lucro e acréscimos nos indicadores de custo, e os confronta com a disponibilidade de equipamentos aperfeiçoados tecnicamente. Dean e Smith (1955) criticam o sistema MAPI por demandar diversas simplificações para sua aplicação. Os mesmos autores acrescentam que o MAPI apóia-se exclusivamente no fluxo de caixa descontado para comparação da alternativa de substituição com outras alternativas de investimento (por exemplo, aluguel do equipamento).

De acordo com Alchian (1958), o desenvolvimento da Programação Dinâmica possibilitou incorporar modelos computacionais aos procedimentos de SE, permitindo análises mais complexas e detalhadas. Merecem destaque ainda os modelos de programação dinâmica de Weingartner (1965) e de Hirohide (1965), também amplamente citados em SE.

\subsubsection{Vida Econômica}

A Vida Econômica de um bem é caracterizada pelo ponto ótimo de substituição, isto é, o ponto em que o custo é mínimo. Dois métodos conceitualmente semelhantes são recomendados para a determinação da Vida Econômica de um equipamento: Valor Anual Uniforme Equivalente (VAUE) ou Custo Anual Uniforme Equivalente (CAUE). Ambos são usados para comparação de projetos com vidas diferentes (ARDALAN, 2000; BLANK, 1998; TARQUIN, 1998; CASAROTTO FILHO; KOPITTKE, 2000; HESS et al., 1973; MOTTA; CALÔBA, 2002; SULLIVAN et al. 2003).

O VAUE é um método de análise de investimentos que trabalha no conceito de valor anual. Esse método é recomendado para comparação de projetos com vidas diferentes, pois utiliza uma base de tempo anual para confrontar as alternativas (CASAROTTO FILHO; KOPITTKE, 2000). O método consiste em transformar um fluxo de caixa irregular em uma série uniforme equivalente, a uma Taxa Mínima de Atratividade (TMA). O CAUE, por sua vez, considera apenas os custos, excluindo as receitas (BLANK, 1998; TARQUIN, 1998; CASAROTTO FILHO; KOPITTKE, 2000).

$O$ Custo Anual $(C A)$ de um equipamento corresponde à soma do Custo de Operação e Manutenção (COM) com o Custo de Recuperação de Capital (CRC). O 
Política para Avaliação e Substituição de Frota por Meio da Adoção de Modelo Multicritério Aray Gustavo Feldens, Claudio José Muller, Tiago Pascoal Filomena, Francisco José Kliemann Neto,

CAUE do período $t$ (em anos) corresponde ao CA acumulado até o ano t menos o valor residual do equipamento. Em geral, as curvas de substituição possuem um comportamento exponencial (COM é representado por uma curva exponencial crescente, ao passo que o CRC é uma exponencial decrescente). O Ponto de Substituição ou Vida Econômica do equipamento ocorre quando:

$$
\frac{\partial C A U E_{t}}{\partial t}=0
$$

Este é o modelo mais simples, considerando uma substituição idêntica com horizonte infinito. Outros modelos são descritos na sequência.

\subsubsection{Modelos de Substituição de Frotas de Veículos}

Algumas considerações precedem a apresentação dos modelos de substituição. $\mathrm{O}$ bem atual (considerado para substituição) é denominado Defensor, enquanto que o novo bem (que pode substituir o atual) é o Desafiante. O horizonte do tempo de análise pode ser definido como finito ou infinito. Quando a necessidade do uso do bem é limitada, a análise é geralmente finita. Quando o uso do bem é por tempo indeterminado, considera-se um horizonte de tempo infinito (HARTMAN, 2005).

Casarotto Filho e Kopittke (2000) inserem os principais modelos de substituição em cinco classes: (i) Baixa sem reposição: não é desejável a substituição do ativo, pois este será mantido apenas enquanto o seu VPL for positivo (i.e., enquanto o ativo for rentável); (ii) Substituição idêntica: determina-se a Vida Econômica do defensor e o ponto de substituição do bem velho por outro bem idêntico novo, buscando-se a minimização do CAUE; (iii) Substituição não-idêntica: compara-se a Vida Econômica do Defensor com a do Desafiante; caso o Desafiante tenha um CAUE menor que o Defensor, parte-se para determinação do ano em que o Defensor deve ser substituído pelo Desafiante; (iv) Substituição com progresso tecnológico: semelhante à substituição não-idêntica, incorpora as vantagens do progresso tecnológico à rentabilidade do Desafiante; e (v) Substituição estratégica: incorporam-se fatores mercadológicos, estratégicos e intangíveis no processo de decisão. 
Política para Avaliação e Substituição de Frota por Meio da Adoção de Modelo Multicritério Aray Gustavo Feldens, Claudio José Muller, Tiago Pascoal Filomena, Francisco José Kliemann Neto, Antenor dos Santos Castro, Michel José Anzanello

Smith (1957) publicou um estudo pioneiro na área de Substituição de Frotas de veículos, avaliando tanto a política ideal como as características ideais dos veículos a serem adquiridos. O mesmo autor enfatiza que somente os custos que variam com o tempo e/ou uso devem ser considerados na determinação da política. Eilon et al. (1966) desenvolveram uma política para uma frota de caminhões enfatizando dois aspectos: (1) determinação da vida econômica do veículo; e (2) utilização de uma política única para toda frota, ou geração de políticas individuais para cada veículo. O trabalho utilizou um modelo linear para o custo de manutenção e um modelo exponencial para o valor residual. Avramovich et al. (1982) descreveram um sistema de apoio à decisão utilizando programação linear para determinar a substituição de uma frota de vans. $O$ modelo indica o mix ideal de substituição e relata o impacto financeiro para a empresa em função da política adotada.

Waddell (1983) utilizou um modelo de programação dinâmica para determinação da política ideal para uma frota de carros de passageiros e caminhões. O modelo incluiu custos de manutenção em função da idade, custos de licenciamento e depreciação. Os veículos foram agrupados em função da quilometragem, idade e tipo. Vemuganti, Oblak e Aggarwal (1989) desenvolveram um modelo de redes utilizando o método Simplex para a substituição de frota. O modelo considera um horizonte finito, tamanho de frota variável e com limite no orçamento anual. Demais modelos de substituição de frota podem ser obtidos em Karabakal et al. (1994); Navon e Maor (1995), Kabir (1996), Scarf e Boamura (1999), Di e Hauke (2000), Knasnabis et al. (2002) e Pinar e Hartman (2004). Os modelos desenvolvidos nestes trabalhos apresentam modelagens matemáticas sofisticadas, mas não consideram múltiplos critérios de decisão e/ou intangíveis para a determinação da política.

\subsection{Apoio à Decisão Multicritério}

Clemen (1996) afirma que existem quatro fontes de dificuldades para a tomada de decisão: complexidade, incerteza, múltiplos objetivos e múltiplas perspectivas. Os critérios de decisão são subjetivamente definidos pelos membros do grupo avaliador (SULLIVAN et al., 2003), e são influenciados pelo tipo de problema e pela intuição de 
Política para Avaliação e Substituição de Frota por Meio da Adoção de Modelo Multicritério Aray Gustavo Feldens, Claudio José Muller, Tiago Pascoal Filomena, Francisco José Kliemann Neto, Antenor dos Santos Castro, Michel José Anzanello

quais critérios podem indicar as diferenças relevantes entre as alternativas de decisão. O número de critérios deve ser equilibrado, pois um elevado número de critérios torna a análise complexa e de difícil visualização, enquanto que poucos critérios limitam a capacidade de discriminação entre as alternativas.

Segundo Keeney e Raiffa (1976), o problema do decisor consiste em avaliar as alternativas possíveis através do balanço das vantagens e desvantagens de um objetivo contra outro. Considerando ai como uma alternativa possível e o conjunto $A$ como todas as alternativas possíveis, cada ai vai gerar um nível de valor xi para um conjunto de atributos X. Como cada xi é, em geral, medido em unidades diferentes, é necessário definir uma função $v$ com a propriedade apresentada na equação (1):

$$
v\left(x_{1}, x_{2}, \ldots, x_{n}\right) \succ v\left(x_{1}{ }^{\prime}, x_{2}{ }^{\prime}, \ldots, x_{n}{ }^{\prime}\right)
$$

O símbolo $\succ$ significa 'preferido ou indiferente a'. Esta função é determinada a partir das preferências do decisor e serve para comparar diferentes atributos indiretamente. O objetivo do decisor passa a ser escolher a do conjunto A para maximizar v. Para cada a existe uma combinação de $x i$, representada pelo vetor $x$. Considerando $x^{\prime}=\left(x 1^{\prime}, x 2^{\prime}, \ldots, x^{\prime}\right)$ e $x^{\prime \prime}=\left(x 1^{\prime \prime}, x 2^{\prime \prime}, \ldots, x n^{\prime \prime}\right)$, diz-se que $x^{\prime}$ domina $x^{\prime \prime}$ quando: (a) xi' $\succ$ xi" para todo i; e, (b) xi' $\succ$ xi" para alguns i (KEENEY; RAIFFA, 1976). Os vetores considerados indiferentes para o decisor estão localizados na mesma Curva de Indiferença. A Curva de Indiferença ou Superfície de Indiferença é determinada pela taxa de substituição do decisor. Cada indivíduo pode ter uma taxa marginal de substituição distinta, dependendo das suas preferências (COPELAND et al., 2005).

\subsubsection{Multiattribute Utility Theory (MAUT)}

Segundo Casarotto Filho e Kopittke (2000), o método MAUT é uma das sistemáticas mais simples para análise multicritério, consistindo na atribuição de pesos aos diversos critérios e notas a cada alternativa, em todos os critérios. O MAUT cria um score ponderado para cada critério, sendo que a alternativa escolhida será aquela que obtiver o maior somatório. Além de ser conceitualmente simples, o MAUT também 
Política para Avaliação e Substituição de Frota por Meio da Adoção de Modelo Multicritério Aray Gustavo Feldens, Claudio José Muller, Tiago Pascoal Filomena, Francisco José Kliemann Neto, Antenor dos Santos Castro, Michel José Anzanello

permite o desenvolvimento de cenários e alterações nos pesos dos critérios sem o uso de ferramentas computacionais sofisticadas. Por outro lado, os resultados dos scores ponderados podem fugir da sensibilidade do decisor e a manipulação sutil dos pesos pode escapar da percepção do decisor.

\subsubsection{Analytical Hierarchy Process (AHP)}

O método AHP é uma ferramenta de auxílio à decisão multicritério que dispensa um escalonamento numérico, visto que as comparações são feitas de forma relativa entre as alternativas, isto é, compara-se a preferência do decisor par-a-par (SAATY, 1990).

O AHP utiliza uma escala de 1 a 9 para os julgamentos comparativos entre as alternativas para cada critério; 1 significa alternativas igualmente importantes e 9 indica que o decisor prefere nove vezes uma alternativa à outra. Uma das vantagens do AHP consiste na sua integração a outras ferramentas de decisão. Encontra-se na literatura o uso do AHP em conjunto com Programação Linear (GHODSYPOUR; O'BRIEN, 1998), Redes Neurais (KUO et al., 2002), Fuzzy Theory (KUO et al., 2002), Data Envelopment Analysis (SARKIS, 1997; YANG; KUO, 2003), Programação por Objetivos (SCHNIEDERJANS; WILSON, 1991) ou mesmo a combinação do AHP com múltiplas ferramentas (KAHRAMAN et al., 2006).

\subsubsection{Modelos AHP/QFD}

O uso combinado do AHP com Quality Function Deployment (QFD) permite a avaliação, identificação e priorização de atributos, demandas e/ou especificações. Ele é particularmente útil para a identificação de preferências do cliente, relacionando estas preferências aos atributos do produto, processo ou serviço. Tais atributos representam especificações técnicas para cada estágio do desenvolvimento do produto e processo (SULLIVAN, 1986).

A combinação AHP/QFD tem sido utilizada em requisitos demandados para casas pré-fabricadas (Armacost et al., 1994); localização de instalações (Chuang, 2001); estratégia de marketing (Lu e Madu, 1994); design de produto (Kwong; Bai, 
Política para Avaliação e Substituição de Frota por Meio da Adoção de Modelo Multicritério Aray Gustavo Feldens, Claudio José Muller, Tiago Pascoal Filomena, Francisco José Kliemann Neto, Antenor dos Santos Castro, Michel José Anzanello

2003) e seleção de workstations (Fogliatto e Guimaraes, 2004), entre outros. Ho et al. (1999) apresentam um modelo que utiliza o Nominal Group Technique (NGT) para capturar as informações da equipe de desenvolvimento e utilizam o AHP/QFD para determinar os critérios e pesos de decisão. No entanto, não foi observado o uso de métodos multicritério integrados a métodos econômicos para substituição de equipamentos, mais especificamente frota. Neste estudo, os Métodos MAUT, AHP e QFD são integrados com dados econômicos para tomada de decisão referente à substituição de ônibus urbanos.

\section{MÉTOdO PARA DESENVOLVIMENTO DE POLÍTICAS DE SBUSTITUIÇÃO DE FROTAS PARA TRANSPORTE PÚBLICO URBANO}

A sistemática proposta está estruturada em quatro etapas. As etapas 2 (Análise Econômica) e 3 (Análise de Critérios Não-Econômicos) podem ser conduzidas em paralelo, visto que estas fornecem subsídios à etapa seguinte. É importante enfatizar que as etapas são descritas com foco nas características de frotas de ônibus, que consiste no alvo deste estudo. A utilização da metodologia em veículos de outra natureza demanda adaptações nas variáveis descritivas deste veículo. O detalhamento de cada etapa é apresentado a seguir.

\subsection{Etapa 1 - Levantamento de dados}

Nesta etapa, o foco é identificar e levantar os principais dados que servirão de base para a análise quantitativa e qualitativa. Os dados devem ser relevantes à descrição da frota e atividades relacionadas, bem como devem oferecer entendimento sobre informações estratégicas e econômicas da empresa. Deve-se priorizar informações relativas aos procedimentos de operação, manutenção e planejamento. No caso de terceirização destes processos, deve-se estudar os processos na empresa terceirizada, bem como detalhes referentes aos contratos desta terceirização.

A frota deve ser caracterizada para a comparação de diferentes tipos de veículos (os quais podem ser decompostos em subfrotas), e é efetivada com base em 
Política para Avaliação e Substituição de Frota por Meio da Adoção de Modelo Multicritério Aray Gustavo Feldens, Claudio José Muller, Tiago Pascoal Filomena, Francisco José Kliemann Neto, Antenor dos Santos Castro, Michel José Anzanello

variáveis descritivas dos componentes do veículo. O ônibus urbano é um conjunto de dois componentes: chassis e carroceria, geralmente adquiridos de fabricantes distintos. O fabricante da carroceria é responsável pela montagem final do chassi na carroceria do ônibus.

A classificação de veículos em subfrotas deve utilizar as seguintes variáveis descritivas: ano de fabricação do ônibus (e ano de início de operação, caso seja diferente), modelo de chassis e modelo de carroceria. Algumas características especiais também devem ser levantadas, principalmente aquelas que afetam significativamente 0 desempenho econômico/operacional (existência e tipo de ar-condicionado, tipo de câmbio, potência do motor, entre outros). É importante considerar que, com o avanço tecnológico dos ônibus, novas características técnicas devem ser consideradas.

Dados de manutenção também devem ser levantados, destacando-se custos de peças e horas de mão-de-obra demandadas. Estes dados serão utilizados para construir os fluxos de caixa para análise econômica e determinação da Vida Econômica. Recomenda-se o mapeamento das demandas de manutenção com base em cada veículo; ou pelo menos por tipo de veículo. A inclusão de dados adicionais sugeridos pela equipe deve ser considerada.

\subsection{Etapa 2 - Análise econômica}

$\mathrm{Na}$ análise econômica, objetiva-se determinar o Custo Anual Equivalente Uniforme (CAUE) de cada subfrota/veículo e a Vida Econômica para Substituição Idêntica. O CAUE é determinado através do fluxo de caixa das subfrotas/veículos ao longo da sua vida útil. Para tanto, coletam-se custos relacionados à aquisição, operação, manutenção e depreciação, bem como o valor de revenda ano-a-ano. É importante também compreender o regime de tributação utilizado na empresa. Normalmente, as empresas de ônibus operam sob o regime de lucro real e o imposto dedutível é aproximadamente $34 \%$, o que inclui a Contribuição Social sobre Lucro Líquido (CSSL), Contribuição para o Financiamento da Seguridade Social (COFINS) e Imposto de Renda da Pessoa Jurídica (IRPJ). 
Política para Avaliação e Substituição de Frota por Meio da Adoção de Modelo Multicritério Aray Gustavo Feldens, Claudio José Muller, Tiago Pascoal Filomena, Francisco José Kliemann Neto, Antenor dos Santos Castro, Michel José Anzanello

A princípio, os valores de custo coletados se encontram no valor nominal. É importante saber o período no qual o valor está baseado, pois o valor registrado pode ser diferente do período em que o item foi utilizado. Por exemplo, a base de dados da empresa pode apresentar o valor atual das peças, mas é necessário conhecer o valor na época em que foram utilizadas. Uma alternativa para o cálculo deste valor consiste na utilização do índice de inflação específico do período anterior até o atual. As seções seguintes descrevem como os custos são calculados.

\subsubsection{Custo de aquisição, valor de revenda e custo de recuperação de capital}

O custo de aquisição do veículo é composto pelo custo do chassi, carroceria e outros equipamentos especiais (ar-condicionado e elevador, entre outros). Tendo-se em vista a utilização de financiamento neste tipo de aquisição, deve-se calcular o VPL do financiamento ao invés de usar o valor de aquisição contábil. O valor de revenda pode ser estimado por dados disponíveis no mercado, julgamento de especialistas ou por um modelo de depreciação. O cálculo do Custo de Recuperação de Capital (CRC) inclui o ônus do Imposto de Renda (IR), presente na venda efetiva. O ônus do IR é a diferença contábil entre o valor efetivo de revenda e o valor contábil do veículo, obedecendo à seguinte regra: paga-se IR quando o valor de revenda é maior que o valor contábil e recupera-se IR quando é menor. As equações (2) à (6) representam as expressões matemáticas utilizadas, sendo que o subíndice t é medido em anos. Os custos são expressos em unidades financeiras atuais.

$$
\begin{gathered}
I_{t}=I_{t-1}-\sum_{t=0}^{t} D R_{t} \\
D C_{t}=T D C^{*} I_{o} \\
O N U S_{t}=\left[\left(1-D C^{*} t\right)^{*} I_{0}-I_{t}\right] * \tau
\end{gathered}
$$


Política para Avaliação e Substituição de Frota por Meio da Adoção de Modelo Multicritério Aray Gustavo Feldens, Claudio José Muller, Tiago Pascoal Filomena, Francisco José Kliemann Neto,

$$
\begin{gathered}
C R C_{t}=I_{t}-I_{t-1} *(1+i)+D C * \tau \\
C R C V_{t}=I_{t}-I_{t-1} *(1+i)+D C * \tau+O N U S_{t}
\end{gathered}
$$

A equação (2) define o valor de mercado do veículo (It) com base na depreciação de mercado (DRt), ao passo que a equação (3) apresenta a depreciação contábil (DCt), onde 10 é o valor de compra original e TDC a taxa de depreciação contábil. Na equação (4) é apresentado o cálculo do ônus da venda do veículo (ONUSt), sendo t a taxa de imposto de renda. A equação (5) calcula o custo de recuperação de capital (CRCt), sendo i a taxa de desconto. Finalmente, a equação (6) fornece o custo de recuperação de capital com a venda do equipamento (CRCVt).

\subsubsection{Custo de operação}

O custo de operação (COt) é apresentado na equação (7), e é composto por custos relacionados ao consumo de combustível (COMBt), consumo de lubrificantes (LUBRt) e material rodante - pneus, câmaras e recapagens - (RODt). Esses custos devem ser expressos na razão custo/km, possibilitando a comparação econômica entre veículos diferentes e do próprio veículo em períodos distintos. Outros gastos (lavagens e licenciamento) podem ser desconsiderados, a menos que sejam significativos, variem com o tempo e/ou uso ou dependam do tipo/características dos ônibus.

$$
C O_{t}=(1-\tau) *\left(C O M B_{t}+L U B R_{t}+R O D_{t}\right)
$$

\subsubsection{Custo de manutenção}

O custo de manutenção (CMt), apresentado na equação (8), é composto pelo custo de mão-de-obra direta de manutenção (MODt), custo em mão-de-obra terceirizada de manutenção (MOTt), custo de horas de equipamentos/instalações 
Política para Avaliação e Substituição de Frota por Meio da Adoção de Modelo Multicritério Aray Gustavo Feldens, Claudio José Muller, Tiago Pascoal Filomena, Francisco José Kliemann Neto, Antenor dos Santos Castro, Michel José Anzanello

utilizadas (EQUIPt), custo em horas imobilizadas (IMOB t) e custo em peças de reposição (PEÇAS t).

$$
C M_{t}=(1-\tau) *\left(M O D_{t}+M O T_{t}+E Q U I P_{t}+I M O B_{t}+P E C ̧ A S_{t}\right)
$$

Custos adicionais podem ser considerados, desde que sejam (i) relevantes ao uso do ônibus, (ii) significativos em termos de valor, e (iii) não-uniformes entre diferentes modelos de ônibus. Ex: a aquisição de um ônibus equipado com um combustível alternativo gera um custo de suprimentos deste combustível (gestão, estoque e risco, entre outros).

\subsubsection{Previsão do Fluxo de Caixa}

A previsão de gastos restringe-se às operações de manutenção de um veículo, visto que os custos operacionais se mantêm estáveis com o tempo (desde que mantidas as condições de rodagem e manutenção). A previsão de gastos envolve os operadores de manutenção, e requer a seleção do modelo de previsão mais adequado. O custo operacional é previsto em unidades de custo $/ \mathrm{km}$.

Os custos de manutenção devem utilizar os valores reais ao invés dos nominais, deflacionando-se os valores. Tal medida é justificada pela distorsão nas previsões causadas por valores elevados de inflação. Recomenda-se utilizar um índice deflator (de preferência um setorial para peças e outro para MOD) para converter os valores nominais para reais.

\subsubsection{Cálculo do CAUE}

O CAUE é determinado através dos custos anuais de manutenção, operação e recuperação de capital, e então utilizado para cálculo da Vida Econômica. As equações (9) a (12) são utilizadas para o cálculo da Vida Econômica, expressa em anos. 
Política para Avaliação e Substituição de Frota por Meio da Adoção de Modelo Multicritério Aray Gustavo Feldens, Claudio José Muller, Tiago Pascoal Filomena, Francisco José Kliemann Neto,

$$
\begin{gathered}
C A_{t}=C R C_{t}+C M_{t}+C O_{t} \\
C A V_{t}=C R C V_{t}+C M_{t}+C O_{t} \\
V P L_{t}=\left(\sum_{t=0}^{t-1} \frac{C A_{t}}{\left(1+i_{n}\right)^{t}}\right)+\frac{C A V_{t}}{\left(1+i_{n}\right)^{t}} \\
C A U E_{t}=V P L_{t} *\left[\frac{i_{r} *\left(1+i_{r}\right)^{t}}{\left(1+i_{r}\right)^{t}-1}\right]
\end{gathered}
$$

A equação (9) apresenta o custo anual do veículo (CAt) com base no custo de recuperação de capital (CRCt), custo de manutenção (CMt) e custo de operação (COt). $\mathrm{Na}$ equação (10) é calculado o custo anual com a venda do equipamento (CAVt), usando-se para isto o custo de recuperação de capital com a venda do equipamento (CRCVt), o custo de manutenção (CMt) e o custo de operação (COt). O valor presente líquido (VPLt), calculado na equação (11), utiliza a taxa de desconto nominal (in), sendo que o custo anual no ano zero é zero $(C A 0=0)$. Nesta mesma equação, pode-se substituir a taxa de desconto nominal (in) pela taxa de desconto real (ir), caso os valores do fluxo de caixa já estejam em valores reais. A equação (12) traz o custo anual uniforme equivalente (CAUEt), sendo utilizado para seu cálculo a taxa de desconto real (ir). Neste estudo, optou-se por ir para evitar eventuais distorções do in sobre o CAUEt.

\subsection{Etapa 3 - Análise de critérios não-economicos}

Esta etapa apresenta uma metodologia multicritério híbrida, através de uma combinação dos métodos MAUT, AHP e QFD (aqui denominado método MAQ), e está baseado na proposta de Fogliatto e Guimarães (2004). Outros métodos, que não o $M A Q$, podem ser utilizados para a análise dos critérios não-econômicos e determinação dos benefícios intangíveis de um tipo de ônibus. As ferramentas utilizadas para execução desta etapa são apresentadas na Figura 1, e descritas nas seções seguintes. 
Política para Avaliação e Substituição de Frota por Meio da Adoção de Modelo Multicritério Aray Gustavo Feldens, Claudio José Muller, Tiago Pascoal Filomena, Francisco José Kliemann Neto, Antenor dos Santos Castro, Michel José Anzanello

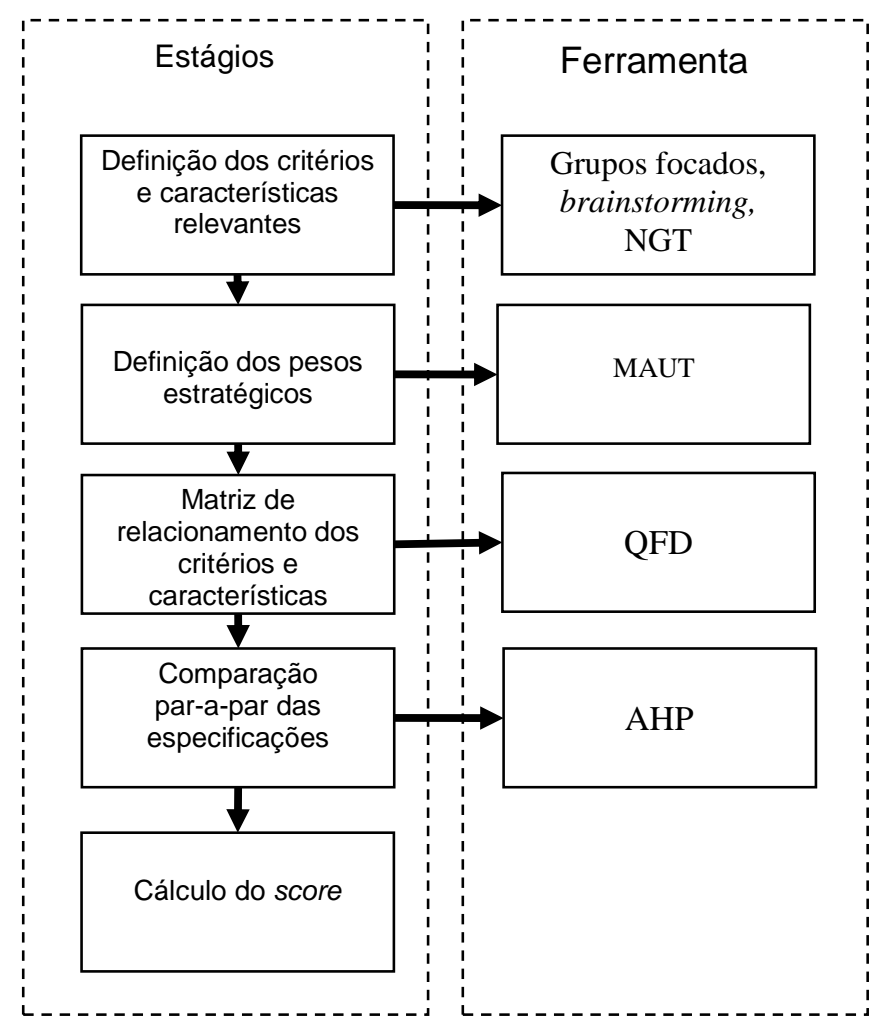

Figura 1: Estrutura do MAQ

O score final para cada especificação do conjunto de características é equivalente a um índice de utilidade (benefício) para as mesmas. O veículo terá o score final definido a partir da soma das especificações que possui. Salienta-se que o método está baseado num modelo aditivo, ou seja, não são consideradas interações entre os diferentes tipos de características.

\subsubsection{Definição dos critérios e características relevantes}

Nesta etapa são definidos os critérios primários e secundários, através da participação de diretores, gerentes e funcionários com conhecimento técnico avançado. Os critérios primários compreendem perspectivas consideradas importantes pela empresa para o seu planejamento estratégico, e devem ser relevantes e exclusivos. Constituem exemplos de critérios primários: operação, manutenção, serviço ao usuário 
Política para Avaliação e Substituição de Frota por Meio da Adoção de Modelo Multicritério Aray Gustavo Feldens, Claudio José Muller, Tiago Pascoal Filomena, Francisco José Kliemann Neto,

e ambiente. Já os critérios secundários são indicadores que medem estas perspectivas, devendo ser representados através de escalas ou unidades quantitativas.

O próximo passo é determinar as características do ônibus a serem incluídas na análise. Os pré-requisitos para inclusão de uma característica são: (i) conflito entre as diferentes partes e/ou perspectivas (critérios) na opção da característica; e/ou (ii) impacto significativo no custo do ônibus. Características dispensáveis/opcionais são representadas por situações onde não há dúvida de opção entre os critérios, e/ou tais características são exigidas por leis ou regulamentos.

Para levantamento de idéias, conceitos e soluções, deve-se utilizar ferramentas de decisão em grupo. Brainstorming, grupos focados e Nominal Group Technique (NGT) são algumas das técnicas recomendadas. O NGT é recomendado para grupos heterogêneos e com participantes de diferentes níveis hierárquicos (CLEMEN, 1996).

\subsubsection{Determinação dos pesos estratégicos (MAUT)}

A ordem de menção de cada valor apontada pelos entrevistados deve ser colocada no inverso de sua respectiva posição; ou seja, 1/aij. Assim, o critério mais importante mencionado pelo entrevistado recebe um peso de importância $1 / 1=1,0$, o segundo mais importante recebe um peso de importância $1 / 2=0,5$, e assim por diante. Esta função inversa garante um peso alto de importância para os primeiros critérios mencionados (Ribeiro et al., 2001). A Tabela 1 ilustra o cálculo dos pesos para M decisores e $\mathrm{N}$ critérios.

Os pesos obtidos são transformados em pesos ponderados para cada grupo de critérios secundários e primários. Para a determinação do peso ponderado de cada critério sobre o restante é realizada uma relativização de seu percentual dentro do grupo a que pertence. Este cálculo é feito separadamente para cada hierarquia de critérios, conforme apresentado na Tabela 2. 
Política para Avaliação e Substituição de Frota por Meio da Adoção de Modelo Multicritério Aray Gustavo Feldens, Claudio José Muller, Tiago Pascoal Filomena, Francisco José Kliemann Neto, Antenor dos Santos Castro, Michel José Anzanello

Tabela 1: Cálculo dos pesos dos critérios

\begin{tabular}{|c|c|c|c|c|}
\hline & Critério $_{1}$ & Critério $_{j}$ & $\ldots$ & Critérion $_{N}$ \\
\hline Decisor 1 & $1 / a_{11}$ & $1 / a_{1 j}$ & $\cdots$ & $1 / a_{1 N}$ \\
\hline Decisor $_{i}$ & $1 / a_{i 1}$ & $1 / a_{i j}$ & $\ldots$ & $\cdots$ \\
\hline : & : & $:$ & $:$ & : \\
\hline Decisor $_{M}$ & $1 / a_{M 1}$ & $1 / a_{M j}$ & $\cdots$ & $1 / a_{M N}$ \\
\hline Total & $t_{1}=\sum_{i=1}^{M} 1 / a_{i 1}$ & $t_{j}=\sum_{i=1}^{M} 1 / a_{i j}$ & $\cdots$ & $\sum_{i=1}^{M} 1 / a_{i N}$ \\
\hline Peso ponderado & $w_{1}=\frac{t_{1}}{\sum_{i=1}^{M} \sum_{j=1}^{N} 1 / a_{i j}}$ & $w_{j}=\frac{t_{j}}{\sum_{i=1}^{M} \sum_{j=1}^{N} 1 / a_{i j}}$ & $\cdots$ & $w_{N}=\frac{t_{N}}{\sum_{i=1}^{M} \sum_{j=1}^{N} 1 / a_{i j}}$ \\
\hline
\end{tabular}

\subsubsection{Matriz de relacionamento dos critérios e características (QFD)}

Nesta etapa parte-se para a construção da matriz de relacionamento (Tabela 2) dos critérios versus características técnicas do ônibus. Sugere-se utilizar a escala recomendada por Ribeiro et al. (2001): neutro (0), fraco (1), médio (3) e forte (9). Escalas alternativas podem ser consideradas, dependendo da natureza da aplicação.

Tabela 2: Matriz de relacionamento do QFD

\begin{tabular}{|c|c|c|c|c|c|}
\hline Critérios Primários & Critérios Secundários & 0 & 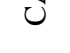 & & \\
\hline & $\mathrm{CS}_{11}$ & $r_{111}$ & $r_{121}$ & $\ldots$ & $\ldots$ \\
\hline $\mathrm{CP}$ & $\mathrm{CS}_{21}$ & $r_{211}$ & $\ldots$ & $\ldots$ & $\ldots$ \\
\hline & $\ldots$ & $\ldots$ & $\ldots$ & $\ldots$ & $\ldots$ \\
\hline & $\mathrm{CS}_{i 1}$ & $\ldots$ & $\ldots$ & $\ldots$ & $r_{i j 1}$ \\
\hline$\ldots$ & $\ldots$ & $\ldots$ & $\ldots$ & $\ldots$ & $\ldots$ \\
\hline & $\mathrm{CS}_{1 k}$ & $r_{11 k}$ & $r_{12 k}$ & $\ldots$ & $\ldots$ \\
\hline $\mathrm{CP}_{2}$ & $\mathrm{CS}_{2 k}$ & $r_{21 k}$ & $\ldots$ & $\ldots$ & $\ldots$ \\
\hline & & $\ldots$ & $\ldots$ & $\ldots$ & \\
\hline & $\mathrm{CS}_{i k}$ & $\ldots$ & $\ldots$ & $\ldots$ & $r_{i j k}$ \\
\hline
\end{tabular}


Política para Avaliação e Substituição de Frota por Meio da Adoção de Modelo Multicritério Aray Gustavo Feldens, Claudio José Muller, Tiago Pascoal Filomena, Francisco José Kliemann Neto, Antenor dos Santos Castro, Michel José Anzanello

A matriz de relacionamento é composta por critérios primários $(\mathrm{CPk})$, critérios secundários referentes a cada critério primário $\left(C S_{i k}\right)$ e características (CARACTJ). Cabe ao decisor estabelecer o nível de relacionamento da característica j com o critério secundário i e critério primário $k\left(r_{i j k}\right)$, valendo-se da escala sugerida acima. Maiores detalhes sobre QFD devem ser buscados em Ribeiro et al. (2001).

\subsubsection{Comparação par-a-par das especificações (AHP)}

Nesta etapa é feita a comparação par-a-par de cada grupo de especificações com cada característica. Recomenda-se a participação de profissionais familiarizados com o equipamento (manutenção e operação) e que detenham conhecimento das especificações de cada característica.

A matriz de julgamento pode ser gerada através de questionário, o qual utiliza uma escala de valores para relacionar as especificações avaliadas. Em uma coluna à esquerda do questionário, listam-se todas as especificações a serem comparadas com as outras especificações na coluna imediatamente à direita. Solicita-se aos entrevistados que indiquem a dominância do elemento da coluna da esquerda sobre o elemento correspondente na mesma linha, na coluna da direita (Tabela 3).

Tabela 3: Matriz de Julgamentos (Adaptado de Saaty, 1990)

\begin{tabular}{|c|c|c|c|c|}
\hline & $\begin{array}{c}\text { Especificação } \\
\mathbf{1}\end{array}$ & Especificação 2 & $\ldots$ & Especificação $\boldsymbol{J}$ \\
\hline Especificação 1 & 1 & $a_{12}$ & $\ldots$ & $a_{1\lrcorner}$ \\
\hline Especificação 2 & $1 / a_{12}$ & 1 & $\ldots$ & $a_{2\lrcorner}$ \\
\hline$\ldots$ & $\ldots$ & $\ldots$ & $\ldots$ & 1 \\
\hline Especificação J & $1 / a_{1 j}$ & $1 / a_{2 x}$ & $\ldots$ & \\
\hline
\end{tabular}

O passo seguinte consiste no cálculo de um vetor de prioridade, dado pelo autovetor máximo normalizado (SAATY, 1991). Dividem-se os elementos de cada coluna pela soma das colunas [conforme a equação (13)], sendo $a_{i j}$ os elementos da Matriz de Julgamento. Então somam-se os elementos em cada linha resultante e dividese esta soma pelo número de elementos na linha [conforme a equação (14)], obtendose o vetor de prioridades $\vec{v}^{(p)}$ (ou seja, a média das colunas normalizadas). 
Política para Avaliação e Substituição de Frota por Meio da Adoção de Modelo Multicritério Aray Gustavo Feldens, Claudio José Muller, Tiago Pascoal Filomena, Francisco José Kliemann Neto, Antenor dos Santos Castro, Michel José Anzanello

$\mathrm{B}=\left[\begin{array}{cccc}a_{11} & a_{12} / \sum_{i=1}^{N} a_{i 2} & & a_{1 J} / \sum_{i=1}^{N} a_{i J} \\ \sum_{21}^{\sum_{i=1}^{N} a_{i 1}} & a_{22}^{N} \sum_{i=1}^{N} a_{i 2} & & a_{2 J}^{N} a_{i J} \\ \vdots & \vdots & : & \vdots \\ a_{i 1} & a_{i 2} / \sum_{i=1}^{N} a_{i 2} & & a_{i J}^{N} a_{i J}\end{array}\right]$

$\overrightarrow{v^{(p)}}=\left[\begin{array}{c}\sum_{j=1}^{N} b_{1 j} \\ N \\ \sum_{j=1}^{N} b_{2 j} \\ N \\ \vdots \\ \frac{\sum_{j=1}^{N} b_{i j}}{N}\end{array}\right]$

Após a determinação das preferências das especificações, é necessário verificar a precisão ou consistência dos resultados obtidos. A precisão é obtida através de uma razão de consistência $(R C)$, sendo que uma razão de consistência menor ou igual a 0,10 é considerada aceitável. Esta razão utiliza o máximo autovalor da Matriz de Julgamentos, $\lambda_{\max }$. Quanto mais próximo $\lambda_{\max }$ for de $n$ (ordem da matriz), mais consistente será o resultado. O índice de consistência $(I C)$ é dado pela equação (15). $\mathrm{RC}$ é apresentada na equação (16). Para tanto, um índice randômico $(I R)$ é gerado de acordo com a ordem da matriz, conforme a Tabela 4.

$$
\begin{aligned}
& I C=\frac{\lambda_{\max }-n}{n-1} \\
& R C=\frac{I C}{I R}
\end{aligned}
$$


Política para Avaliação e Substituição de Frota por Meio da Adoção de Modelo Multicritério Aray Gustavo Feldens, Claudio José Muller, Tiago Pascoal Filomena, Francisco José Kliemann Neto, Antenor dos Santos Castro, Michel José Anzanello

Tabela 4: Índice Randômico para cálculo da razão de consistência $(R C)$

\begin{tabular}{|c|c|c|c|c|c|c|c|c|c|c|c|c|c|c|c|}
\hline $\begin{array}{c}\text { Ordem } \\
\text { da } \\
\text { Matriz }\end{array}$ & $\mathbf{1}$ & $\mathbf{2}$ & $\mathbf{3}$ & $\mathbf{4}$ & $\mathbf{5}$ & $\mathbf{6}$ & $\mathbf{7}$ & $\mathbf{8}$ & $\mathbf{9}$ & $\mathbf{1 0}$ & $\mathbf{1 1}$ & $\mathbf{1 2}$ & $\mathbf{1 3}$ & $\mathbf{1 4}$ & $\mathbf{1 5}$ \\
\hline $\mathrm{IR}$ & $\begin{array}{c}0,0 \\
0\end{array}$ & $\begin{array}{c}0,0 \\
0\end{array}$ & $\begin{array}{c}0,5 \\
8\end{array}$ & $\begin{array}{c}0,9 \\
0\end{array}$ & $\begin{array}{c}1,1 \\
2\end{array}$ & $\begin{array}{c}1,2 \\
4\end{array}$ & $\begin{array}{c}1,3 \\
2\end{array}$ & $\begin{array}{c}1,4 \\
1\end{array}$ & $\begin{array}{c}1,4 \\
5\end{array}$ & $\begin{array}{c}1,4 \\
9\end{array}$ & $\begin{array}{c}1,5 \\
1\end{array}$ & $\begin{array}{c}1,4 \\
8\end{array}$ & $\begin{array}{c}1,5 \\
6\end{array}$ & $\begin{array}{c}1,5 \\
7\end{array}$ & $\begin{array}{c}1,5 \\
9\end{array}$ \\
\hline
\end{tabular}

Fonte: Saaty (2008).

Se a razão de consistência for satisfatória, o vetor de prioridades corresponderá aos pesos das preferências das especificações. Caso não seja, deve-se preencher a matriz de julgamentos novamente, identificando os julgamentos que apresentaram maior inconsistência.

\subsubsection{Cálculo do score}

Considerando $C$ critérios primários, $A C$ critérios secundários, $B$ características e $B D$ especificações, o cálculo do benefício (utilidade) do equipamento, $B\left(E S P_{11}, \ldots\right.$, $\left.E S P_{C D}\right)$, é dado pela equação (17).

$$
B\left(E S P_{11}, \ldots, E S P_{C D}\right)=\sum_{i=1}^{A} \sum_{j=1}^{B} \sum_{k=1}^{C} \sum_{x=1}^{D} w c p_{k} * w c s_{i k} * r_{i j k} * p\left(E S P_{j x}\right)
$$

Para tanto, utiliza-se o peso normalizado do critério primário $k\left(w c p_{k}\right)$, peso normalizado do critério secundário $i$ (wcsik), força de relacionamento entre a característica $j$ e o critério secundário $i\left(r_{i j k}\right)$, e peso das preferências de especificação $x$ da característica $j\left(p\left(E S P_{j x}\right)\right)$. Outras informações relevantes podem ser incluídas na análise, como exigências regulatórias, fatores implícitos e exigências de especificações, entre outros.

\subsection{Etapa 4 - Análise benefício/custo}

A etapa de Análise Benefício/Custo consiste em contrabalançar o custo econômico (Análise Econômica) e os benefícios intangíveis (Análise dos Critérios Não- 
Política para Avaliação e Substituição de Frota por Meio da Adoção de Modelo Multicritério Aray Gustavo Feldens, Claudio José Muller, Tiago Pascoal Filomena, Francisco José Kliemann Neto, Antenor dos Santos Castro, Michel José Anzanello

Econômicos) do veículo. Deve-se utilizar o CAUE mínimo para que a comparação seja justa entre equipamentos com Vida Econômica em períodos diferentes. Caso se compare o CAUE por $\mathrm{km}$ e/ou por passageiro, recomenda-se o uso de uma quilometragem padronizada, além de considerar a mesma taxa de ocupação ou número de passageiros médio por km, evitando distorções.

O decisor deve determinar o peso que será atribuído ao custo e aos benefícios; este peso indica o quanto ele está disposto a pagar pelos benefícios proporcionados pelo equipamento. A taxa de substituição do decisor é ilustrada na Figura 2, que traz a análise de fronteira relacionando benefício e custo. Os equipamentos 1, 2 e 3 estão localizados na fronteira eficiente, sendo listados como possíveis soluções para o problema.

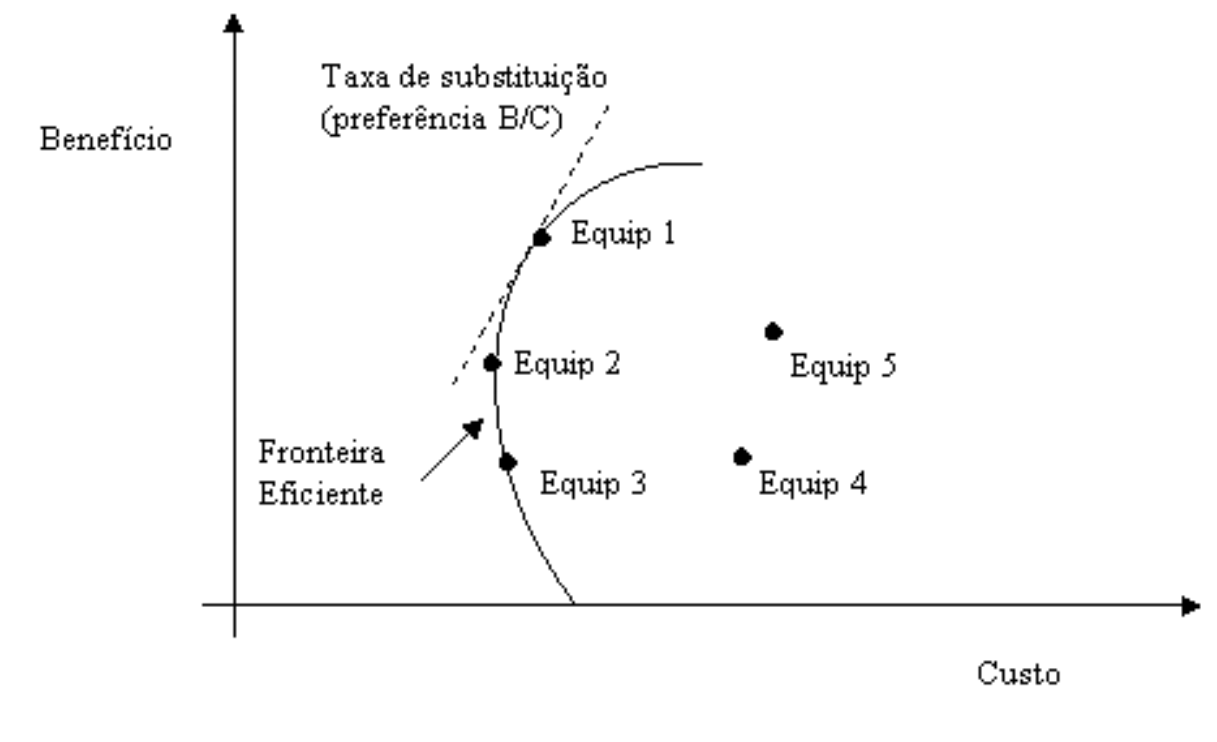

Figura 2: Benefício/Custo com diferentes taxas de substituição

O Benefício/Custo (Ben/Cus) é dado pela equação (18). O parâmetro $\rho(0<\rho<1)$ é a importância do decisor em relação ao benefício e custo $\rho$ igual à 0,5 significa pesos iguais para benefício e custo, enquanto que $\rho>0,5$ sinaliza maior peso ao benefício. $O$ parâmetro $\rho$ não inclui os valores 0 e 1 , visto que o estudo prima pela interação de custo e benefício, e não pela análise restrita de um desses componentes. É importante 
Política para Avaliação e Substituição de Frota por Meio da Adoção de Modelo Multicritério Aray Gustavo Feldens, Claudio José Muller, Tiago Pascoal Filomena, Francisco José Kliemann Neto, Antenor dos Santos Castro, Michel José Anzanello

mencionar que o CAUE mínimo (CAUE na Vida Econômica) deve ser utilizado para a comparação justa entre equipamentos com vidas diferentes.

$$
\operatorname{Ben} / \mathrm{Cus}=\frac{\rho * B e n}{(1-\rho) * C A U E_{\min }}
$$

Finalmente, a política de substituição de frota da empresa pode ser determinada com base no cálculo do CAUE, dos benefícios e do índice Ben/Cus. Complementarmente, deve-se definir se essa política será idêntica para toda frota, ou se existirá uma política por subfrota ou mesmo por veículo. Recomenda-se a revisão anual da política para verificar quais veículos devem ser substituídos nos anos seguintes, bem como para a atualização dos critérios econômicos e não-econômicos, caso necessário.

\section{APLICAÇÃO DO MODELO}

O modelo proposto foi aplicado em uma empresa de transporte público urbano por ônibus. Por questões estratégicas da empresa e limitações de espaço, o caso prático foi resumido à apresentação dos principais resultados. Os valores referentes à parte econômica foram modificados, mas a relação de grandeza entre as frotas foi mantida.

A frota da empresa foi classificada em subfrotas de acordo com as seguintes variáveis descritivas: ( $I$ ) carroceria (marca e modelo), (ii) motor (marca e modelo), (iii) idade (a partir da data de circulação inicial), (iv) ar-condicionado (com ou sem), (v) articulado (sim ou não), ( $v i)$ câmbio (manual ou automático), e (vii) piso (alto ou baixo). Foram geradas 11 subfrotas.

A avaliação econômica seguiu os passos definidos na seção 3 , resultando nos critérios econômicos apresentados na Tabela 5. A primeira coluna da Tabela 5 apresenta a identificação da subfrota, enquanto que a segunda traz a idade da subfrota, em anos. Na terceira coluna é apresentado o CAUE mínimo normalizado pela quilometragem, ao passo que a quarta coluna apresenta o CAUE mínimo normalizado 
Política para Avaliação e Substituição de Frota por Meio da Adoção de Modelo Multicritério Aray Gustavo Feldens, Claudio José Muller, Tiago Pascoal Filomena, Francisco José Kliemann Neto, Antenor dos Santos Castro, Michel José Anzanello

pela quilometragem e capacidade de passageiros. A última coluna traz a faixa de troca calculada para cada uma das subfrotas, em anos. A faixa de troca é gerada através da comparação do CAUE/km e do CAUE/km mínimo, sendo que uma diferença superior a $2,5 \%$ indica necessidade de troca. A opinião de especialistas foi incorporada ao processo decisório para definição do ponto de corte.

Tabela 5: Resumo dos critérios da avaliação econômica

\begin{tabular}{|c|c|c|c|c|}
\hline Subfrota & $\begin{array}{c}\text { Idade da } \\
\text { Subfrota } \\
\text { no final de } \\
\mathbf{2 0 0 4} \\
\text { (anos) }\end{array}$ & $\begin{array}{c}\text { CAUE/km } \\
\text { Mínimo }\end{array}$ & $\begin{array}{c}\text { CAUE por } \\
\mathbf{k m} \text { por } \\
\text { passageiro } \\
\mathbf{( R \mathbf { R } / \mathbf { k m }}\end{array}$ & $\begin{array}{c}\text { Faixa } \\
\text { de } \\
\text { Troca } \\
\text { (anos) }\end{array}$ \\
\hline 1 & 10 & 2,0437 & 1,5366 & $9-10$ \\
\hline 2 & 9 & 0,8477 & 1,1302 & $5-10$ \\
\hline 3 & 9 & 0,8967 & 1,1940 & $6-10$ \\
\hline 4 & 8 & 0,8702 & 1,1375 & $4-10$ \\
\hline 5 & 7 & 0,9510 & 1,2391 & $3-10$ \\
\hline 6 & 7 & 1,8641 & 1,4095 & $4-10$ \\
\hline 7 & 5 & 1,5566 & 2,0414 & $2-3$ \\
\hline 8 & 6 & 0,9775 & 1,2374 & $2-3$ \\
\hline 9 & 6 & 1,7539 & 1,3287 & $4-10$ \\
\hline 10 & 6 & 1,4032 & 1,7819 & $2-5$ \\
\hline 11 & 4 & 1,5045 & 1,9757 & $2-3$ \\
\hline
\end{tabular}

Os critérios não-econômicos foram definidos através do método MAQ (ver seção 3.3) e apresentados na Tabela 6. A segunda e terceira coluna apresentam os pesos gerados pelo método; o peso de importância associado ao chassi é $65 \%$ e à carroceria $35 \%$, valores esses definidos com base na opinião de especialistas da empresa. $\mathrm{Na}$ quarta e quinta colunas são apresentados os pesos normalizados em relação ao maior valor de peso do chassi e carroceria, respectivamente. A sexta coluna apresenta o benefício total de cada frota, enquanto que a sétima coluna traz os valores de benefício total normalizados em relação ao maior benefício total. 
Política para Avaliação e Substituição de Frota por Meio da Adoção de Modelo Multicritério Aray Gustavo Feldens, Claudio José Muller, Tiago Pascoal Filomena, Francisco José Kliemann Neto, Antenor dos Santos Castro, Michel José Anzanello

Tabela 6: Resumo dos critérios da avaliação não-econômica

\begin{tabular}{|c|c|c|c|c|c|c|}
\hline Subfrota & $\begin{array}{c}\text { Peso } \\
\text { Chassi } \\
\mathbf{( 6 5 \% )}\end{array}$ & $\begin{array}{c}\text { Peso } \\
\text { Carroceria } \\
\mathbf{( 3 5 \% )}\end{array}$ & $\begin{array}{c}\text { Peso Chassi } \\
\text { Normalizado }\end{array}$ & $\begin{array}{c}\text { Peso } \\
\text { Carroceria } \\
\text { Normalizado }\end{array}$ & $\begin{array}{c}\text { Benefício } \\
\text { Total }\end{array}$ & $\begin{array}{c}\text { Benefício Total } \\
\text { Normalizado }\end{array}$ \\
\hline 1 & 165,73 & 7,00 & $84,96 \%$ & $70,00 \%$ & $79,73 \%$ & $81,59 \%$ \\
\hline 2 & 81,85 & 7,00 & $41,96 \%$ & $70,00 \%$ & $51,77 \%$ & $52,98 \%$ \\
\hline 3 & 81,85 & 7,00 & $41,96 \%$ & $70,00 \%$ & $51,77 \%$ & $52,98 \%$ \\
\hline 4 & 81,85 & 7,00 & $41,96 \%$ & $70,00 \%$ & $51,77 \%$ & $52,98 \%$ \\
\hline 5 & 168,77 & 8,00 & $86,52 \%$ & $80,00 \%$ & $84,24 \%$ & $86,21 \%$ \\
\hline 6 & 165,73 & 9,00 & $84,96 \%$ & $90,00 \%$ & $86,73 \%$ & $88,75 \%$ \\
\hline 7 & 195,06 & 3,00 & $100,00 \%$ & $30,00 \%$ & $75,50 \%$ & $77,26 \%$ \\
\hline 8 & 188,21 & 10,00 & $96,49 \%$ & $100,00 \%$ & $97,72 \%$ & $100,00 \%$ \\
\hline 9 & 165,73 & 9,00 & $84,96 \%$ & $90,00 \%$ & $86,73 \%$ & $88,75 \%$ \\
\hline 10 & 162,69 & 6,00 & $83,40 \%$ & $60,00 \%$ & $75,21 \%$ & $76,97 \%$ \\
\hline 11 & 156,03 & 5,00 & $79,99 \%$ & $50,00 \%$ & $69,49 \%$ & $71,12 \%$ \\
\hline
\end{tabular}

Percebe-se, com base na Tabela 6, que a frota 8 possui o melhor benefício com base no método MAQ. A Tabela 7 apresenta um resumo do benefício/custo de cada frota gerado pela equação (18), utilizando-se $\square=0,5$.

As subfrotas 5 e 8 apresentam a melhor relação Benefício/Custo, ao passo que as subfrotas 7 e 11 demonstram a pior relação Benefício/Custo, conforme apresentado na Tabela 7. É importante ressaltar que as subfrotas 1, 6 e 9 são compostas por veículos articulados. Estes veículos apresentaram uma boa relação Benefício/Custo por conta da taxa de ocupação considerada (75\%). Sabe-se, no entanto, que veículos articulados somente atendem a este nível de serviço em determinados horários e operando em linhas específicas da empresa. De tal forma, a relação Benefício/Custo de um veículo articulado depende de sua alocação a linhas de longo percurso e com altos índices de demanda em várias faixas de horário. 
Política para Avaliação e Substituição de Frota por Meio da Adoção de Modelo Multicritério Aray Gustavo Feldens, Claudio José Muller, Tiago Pascoal Filomena, Francisco José Kliemann Neto,

Antenor dos Santos Castro, Michel José Anzanello

Tabela 7: Resumo do benefício/custo obtido

\begin{tabular}{|c|c|c|}
\hline \multirow{2}{*}{ Subfrota } & Benefício/Custo & Faixas \\
\hline 8 & $100,00 \%$ & \multirow{2}{*}{ Ótimo $100 \%-85 \%$} \\
\hline 5 & $86,66 \%$ & \\
\hline 9 & $82,62 \%$ & \multirow{2}{*}{ Bom $85 \%-60 \%$} \\
\hline 6 & $77,70 \%$ & \\
\hline 1 & $65,53 \%$ & \\
\hline 4 & $57,52 \%$ & \multirow{2}{*}{ Fraco $60 \%-50 \%$} \\
\hline 2 & $54,97 \%$ & \\
\hline 3 & $54,77 \%$ & \\
\hline 10 & $53,91 \%$ & \\
\hline 7 & $48,49 \%$ & \multirow{2}{*}{ Ruim $<50 \%$} \\
\hline 11 & $44,98 \%$ & \\
\hline
\end{tabular}

\section{CONCLUSÕES}

Este trabalho apresentou uma sistemática para definição da melhor política de substituição de frotas de ônibus para transporte coletivo urbano, integrando análises da engenharia econômica (aspectos econômicos) a ferramentas de análise multicritério (aspectos não-econômicos). Aspectos econômicos foram norteados por indicadores pertinentes e fluxos de caixa, valendo-se para isso de custos associados à aquisição e manutenção da frota. Aspectos não-econômicos abordaram questões estratégicas intangíveis por intermédio dos métodos AHP, MAUT e QFD.

O estudo demonstrou que existe uma faixa ideal de troca do veículo, obtida pela análise integrada do CAUE, ferramentas multicritério e incorporação da opinião de especialistas. No entanto, ao prolongar muito esta faixa, o ganho econômico de troca do veículo é reduzido. Dentro desta faixa, devem-se incluir outros aspectos que podem influenciar o momento da troca, como disponibilidade de linhas de financiamento a juro reduzido (o que pode antecipar uma troca de veículo), entre outros. Critérios técnicos (dimensionamento e oferta de serviço), financeiros (linhas de financiamento e saúde financeira da empresa) e regulatórios também podem ajudar a determinar o momento exato da substituição do ônibus. 
Política para Avaliação e Substituição de Frota por Meio da Adoção de Modelo Multicritério Aray Gustavo Feldens, Claudio José Muller, Tiago Pascoal Filomena, Francisco José Kliemann Neto, Antenor dos Santos Castro, Michel José Anzanello

Sugestões para trabalhos futuros incluem a utilização de um modelo estocástico nos fluxos de caixa e também na análise multicritério (visto que o presente estudo utilizou modelos determinísticos). Avalia-se ainda a aplicação de Projetos de Experimentos (MONTGOMERY, 1991) visando identificar a sensibilidade das variáveis de resposta em relação às de entrada e as interações entre os fatores de entrada. A estratificação adicional das subfrotas, de acordo com a diferença do custo de manutenção entre veículos da mesma subfrota, também deverá ser testada. Por fim, a incorporação de custos ambientais na política de substituição (por exemplo, um veículo menos poluente pode antecipar a substituição de um equipamento usado) também será considerada.

\section{REFERÊNCIAS}

ALCHIAN, A. (1958). Economic Replacement Policy: an abbreviated version of R-224. (1 ed.). California, RAND Corporation, $29 \mathrm{p}$.

ARDALAN, A. (2000). Economic \& Financial Analysis for Engineering \& Project Management. Lancaster, Technomic Publishing.

ARMACOST, R.; COMPONATION, P.; MULLENS, M.; SWART, W. (1994). AHP framework for prioritizing customer requirements in QFD: An industrialized housing application. IIE Transactions, 26(4): 72-79.

AVRAMOVICH, D.; COOK, T; LANGSTON, G.; SUTHERLAND, F. A. (1982). Decision Support System for Fleet Management: A Linear Programming Approach. Interfaces, 12(3): 1-9.

BLANK, L.; TARQUIN, A. (1998). Engineering Economy. (4 ed.). McGraw-Hill series in industrial engineering and management science, $722 \mathrm{p}$.

CARTER, W. (1992). To Invest in New Technology or Not? New Tools for Making the Decision. Journal of Accountancy, 173(5): 58.

CASAROTTO FILHO, N.; KOPITTKE, B. (2000). Análise de investimentos: matemática financeira, engenharia econômica, tomada de decisão e estratégia empresarial. (9ed.). São Paulo, Atlas. 
Política para Avaliação e Substituição de Frota por Meio da Adoção de Modelo Multicritério Aray Gustavo Feldens, Claudio José Muller, Tiago Pascoal Filomena, Francisco José Kliemann Neto, Antenor dos Santos Castro, Michel José Anzanello

CHUANG, P. (2001). Combining the Analytic Hierarchy Process and Quality Function Deployment for a Location Decision from a Requirement Perspective. The International Journal of Advanced Manufacturing Technology, 18(11): 842-849.

CLEMEN, R. (1996). Making Hard Decisions: an introduction to decision analysis. (2 ed.). Belmont, Duxbury Press.

COLLAN, M.; LIU, S. (2003). Fuzzy logic and intelligent agents: Towards the next step of capital budgeting decision support. Industrial Management + Data Systems, 103(5/6): 410.

COPELAND, T.; WESTON, J.; SHASTRI, K. (2005). Financial Theory and Corporate Policy. (4 ed.). USA, Pearson Addison Wesley.

DEAN, J.; SMITH, W. (1955). Has MAPI a Place in a Comprehensive System of Capital Controls? The Journal of Business (pre-1986), 28(4): 261.

DI, J.; HAUKE, L. (2000). Optimal fleet utilization and replacement. Transportation Research Part E, 36E(1): 3.

EILON, S.; KING, J.; HUTCHINSON, D. (1996). A Study in Equipment Replacement. Operational Research Quartely, 17(1): 59-71.

EROL, I.; FERRELL, W. (2003). A methodology for selection problems with multiple, conflicting objectives and both qualitative and quantitative criteria. International Journal of Production Economics, 88(3): 187.

FELDENS, A. G. (2006). Sistemática para desenvolvimento de políticas de substituição de frotas de ônibus para transporte público: uma abordagem multicritério. (Dissertação de Mestrado). Programa de Pós-Graduação em Engenharia de Produção. Universidade Federal do Rio Grande do Sul - UFRGS, Porto Alegre.

FLEISCHER, G. (1973). Teoria da aplicação do capital: um estudo das decisões de investimento. (1 ed.). São Paulo, Edgard Blücher, 272 p.

FOGLIATTO, F.; GUIMARAES, L. (2004). User-oriented method for selecting workstation components. International Journal of Industrial Ergonomics, 33(2): 133-147.

GHODSYPOUR, S.; O'BRIEN, C. (1998). A decision support system for supplier selection using an integrated analytic hierarchy process and linear programming. International Journal of Production Economics, 56/57(3): 199-212.

HARTMAN, J. (2005). A note on a strategy for optimal equipment replacement. Production Planning \& Control, 16(7): 733-739. 
Política para Avaliação e Substituição de Frota por Meio da Adoção de Modelo Multicritério Aray Gustavo Feldens, Claudio José Muller, Tiago Pascoal Filomena, Francisco José Kliemann Neto, Antenor dos Santos Castro, Michel José Anzanello

HESS, G. (1973). Engenharia Econômica. (4 ed.). Rio de Janeiro, Forum, 100 p.

HIROHIDE, H. (1965). Capacity Expansion with Facilities under Technological Improvement. Management Science (pre-1986), 11(5), p. 581.

HO, S.; LAI, Y.; CHANG, S. (1999). An integrated group decision-making approach to quality function deployment. IIE Transactions, 31(6): 553-567.

HOTELLING, H. A. (1925). General Mathematical Theory of Depreciation. Journal of the American Statistical Association, 20(151): 340-353.

KABIR, A. (1996). Evaluation of overhaul/replacement policy for a fleet of buses. Journal of Quality in Maintenance Engineering, 2(3): 49.

KAHRAMAN, C.; ERTAY, T.; ZKAN, G. (2006). A fuzzy optimization model for QFD planning process using analytic network approach. European Journal of Operational Research, 171(2): 390-411.

KAPLAN, R. (1986). Must CIM be justified by faith alone? Harvard Business Review, 64(2): 87-95.

KARABAKAL, N.; LOHMANN, J.; BEAN, J. (1994). Parallel replacement under capital rationing constraints. Management Science, 40(3): 305-319.

KEENEY, R.; RAIFFA, H. (1976). Decisions with multiple objectives: preferences and value tradeoffs. (1 ed.). New York, John Wiley \& Sons Inc., 569 p.

KHASNABIS, S.; ALSAIDI, E.; ELLIS, R. (2002). Optimal allocation of resources to meet transit fleet requirements. Journal of Transportation Engineering, 128(6): 509-518.

KUO, R.; CHI, S.; KAO, S. (2002). A decision support system for selecting convenience store location through integration of fuzzy AHP and artificial neural network. Computers in Industry, 47(2): 199-214.

KWONG, C.; BAI, H. (2003). Determining the importance weights for the customer requirements in QFD using a fuzzy AHP with an extent analysis approach. IIE Transactions, 35(7): 619-626.

LU, M.; MADU, C. (1994). Integrating QFD, AHP and benchmarking in strategic marketing. Journal of Business \& Industrial Marketing, 9(1): 41-50.

MONTGOMERY, D. (1991). Design and analysis of experiments. (3 ed.). New York, John Wiley and Sons. 
Política para Avaliação e Substituição de Frota por Meio da Adoção de Modelo Multicritério Aray Gustavo Feldens, Claudio José Muller, Tiago Pascoal Filomena, Francisco José Kliemann Neto, Antenor dos Santos Castro, Michel José Anzanello

MOTTA, R.; CALÔBA, G. (2002). Análise de investimentos: tomada de decisão em projetos industriais. (1 ed.). São Paulo, Atlas, $391 \mathrm{p}$.

NAVON, R.; MAOR, D. (1995). Equipment replacement and optimal size of a civil engineering fleet. Construction Management \& Economics, 13(2): 173-183.

PINAR, K.; HARTMAN, J. (2004). Case Study: Bus Fleet Replacement. The Engineering Economist, 49(3): 253-278.

RIBEIRO, J.; ECHEVESTE, M.; DANILEVICZ, A. (2001). A utilização do QFD na otimização de produtos, processos e serviços. (1 ed.). Porto Alegre, FEENG/UFRGS, $96 \mathrm{p}$.

ROY, B.; VINCKE, P. (1983). Multicriteria analysis: survey and new directions. European Journal of Operational Research, 8(3): 207-218.

SAATY, T. (1991). Método de Análise Hierárquica. (1 ed.). São Paulo, McGraw-Hill, 367 p.

SAATY, T. (1990). How to Make a Decision: The Analytic Hierarchy Process. European Journal of Operational Research, 48(1): 9.

SARKIS, J. (1997). Evaluating flexible manufacturing systems alternatives using data envelopment analysis. The Engineering Economist, 43(1): 25-47.

SCARF, P.; BOUAMRA, O. (1999). A capital equipment replacement model for a fleet with variable size. Journal of Quality in Maintenance Engineering, 5(1): 40.

SCHNIEDERJANS, M.; WILSON, R. (1991). Using the analytic hierarchy process and goal programming for information system project selection. Information \& Management, 20(5): 333-342.

SULLIVAN, L. (1986). Quality Function Deployment. Quality Progress, 19(6): 39-50.

SULLIVAN, W.; WICKS, E.; LUXHOJ, J. (2003). Engineering Economy. (12 ed.). New Jersey: Pearson Education, 675p.

TAYLOR, J. (1923). A Statistical Theory of Depreciation. Journal of the American Statistical Association, 18(144): 1010-1023.

VEMUGANTI, R.; OBLAK, M.; AGGARWAL, A. (1989). Network Models For Fleet Management. Decision Sciences, 20(1): 182-197.

WADDELL, R. A. (1983). Model for Equipment Replacement Decisions and Policies. Interfaces, 13(4): 1-7. 
Política para Avaliação e Substituição de Frota por Meio da Adoção de Modelo Multicritério Aray Gustavo Feldens, Claudio José Muller, Tiago Pascoal Filomena, Francisco José Kliemann Neto, Antenor dos Santos Castro, Michel José Anzanello

WEINGARTNER, H. (1965). Equipment Replacement Analysis. Industrial Management Review (pre-1986), 7(1): 45.

YANG, T.; KUO, C. (2003). A hierarchical AHP/DEA methodology for the facilities layout design problem. European Journal of Operational Research, 147(1): 128-136.

Data de Submissão: 15/07/2009

Data de Aceite: 23/03/2010 